\title{
Serum transferrin as a biomarker of hepatocyte nuclear factor 4 alpha activity and hepatocyte function in liver diseases
}

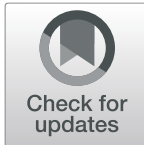

\author{
Nurdan Guldiken ${ }^{1 \dagger}$, Josepmaria Argemi ${ }^{2,3 \dagger}$, Berivan Gurbuz' ${ }^{1}$ Stephen R. Atkinson ${ }^{4}$, Martin Oliverius ${ }^{5}$, Petr Fila ${ }^{5}$, \\ Karim Hamesch ${ }^{1}$, Tony Bruns ${ }^{1}$, Joaquín Cabezas ${ }^{6,7}$, Juan J. Lozano ${ }^{8}$, Jelena Mann ${ }^{9}$, Sheng Cao ${ }^{10}$, \\ Philippe Mathurin ${ }^{11}$, Vijay H. Shah ${ }^{10}$, Christian Trautwein ${ }^{1}$, Mark R. Thursz ${ }^{4}$, Ramon Bataller ${ }^{2}$ and Pavel Strnad ${ }^{1 *}$
}

\begin{abstract}
Background: Serum transferrin levels represent an independent predictor of mortality in patients with liver failure. Hepatocyte nuclear factor 4 alpha (HNF4a) is a master regulator of hepatocyte functions. The aim of this study was to explore whether serum transferrin reflects HNF4a activity.

Methods: Factors regulating transferrin expression in alcoholic hepatitis (AH) were assessed via transcriptomic/ methylomic analysis as well as chromatin immunoprecipitation coupled to DNA sequencing. The findings were corroborated in primary hepatocytes. Serum and liver samples from 40 patients with advanced liver disease of multiple etiologies were also studied.

Results: In patients with advanced liver disease, serum transferrin levels correlated with hepatic transferrin expression ( $r=0.51, p=0.01$ ). Immunohistochemical and biochemical tests confirmed reduced HNF4a and transferrin protein levels in individuals with cirrhosis. In AH, hepatic gene-gene correlation analysis in liver transcriptome revealed an enrichment of HNF4a signature in transferrin-correlated transcriptome while transforming growth factor beta 1 (TGF $\beta 1$ ), tumor necrosis factor a (TNFa), interleukin 1 beta (IL-1 $\beta$ ), and interleukin 6 (IL-6) negatively associated with transferrin signature. A key regulatory region in transferrin promoter was hypermethylated in patients with AH. In primary hepatocytes, treatment with TGF $\beta 1$ or the HNF4a inhibitor BI6015 suppressed transferrin production, while exposure to TNFa, IL-1 $\beta$, and IL-6 had no effect. The correlation between hepatic HNF4A and transferrin mRNA levels was also seen in advanced liver disease.
\end{abstract}

Conclusions: Serum transferrin levels constitute a prognostic and mechanistic biomarker. Consequently, they may serve as a surrogate of impaired hepatic HNF4a signaling and liver failure.

Keywords: End-stage liver disease, Cirrhosis, Alcoholic hepatitis, Transferrin, HNF4alpha

\footnotetext{
* Correspondence: pstrnad@ukaachen.de

${ }^{\dagger}$ Nurdan Guldiken and Josepmaria Argemi contributed equally to this work.

'Department of Internal Medicine III, University Hospital RWTH Aachen,

Pauwelsstraße 30, 52074 Aachen, Germany

Full list of author information is available at the end of the article
}

(c) The Author(s). 2021 Open Access This article is licensed under a Creative Commons Attribution 4.0 International License, which permits use, sharing, adaptation, distribution and reproduction in any medium or format, as long as you give appropriate credit to the original author(s) and the source, provide a link to the Creative Commons licence, and indicate if changes were made. The images or other third party material in this article are included in the article's Creative Commons licence, unless indicated otherwise in a credit line to the material. If material is not included in the article's Creative Commons licence and your intended use is not permitted by statutory regulation or exceeds the permitted use, you will need to obtain permission directly from the copyright holder. To view a copy of this licence, visit http://creativecommons.org/licenses/by/4.0/ The Creative Commons Public Domain Dedication waiver (http://creativecommons.org/publicdomain/zero/1.0/) applies to the data made available in this article, unless otherwise stated in a credit line to the data. 


\section{Background}

The liver is the largest gland of the human body, and accordingly, liver hepatocytes synthesize the majority of serum proteins [1]. Accordingly, the levels of these proteins are diminished in advanced liver disease as they reflect the loss in hepatocytes [1]. Apart from that, the protein synthesis within hepatocytes is subject to a complex regulation by liver-enriched transcription factors (LETFs). Hepatocyte nuclear factors constitute prototypical LETFs and regulate the production of multiple liver-specific proteins [2, 3]. During stress situations, various cytokines such as interleukins 1 and 6 or tumor necrosis factor $\alpha(\mathrm{TNF} \alpha)$ induce the synthesis of species known as acute-phase proteins (APPs) and diminish the production of negative APPs $[1,4]$. Albumin or transferrin are characteristic negative APPs $[1,5]$. Serum levels of negative APPs constitute attractive biomarkers reflecting both the hepatocellular mass and the amount of superimposed systemic stress or inflammation $[1,6]$. In particular, transferrin emerges as a mortality predictor in sepsis and multiple liver disorders $[1,6,7]$.

A defective hepatocyte nuclear factor 4 alpha (HNF4 $\alpha$ )-dependent gene expression was identified as a driver of hepatocellular failure $[8,9]$.

Transferrin is one the most abundant serum proteins produced and secreted by the hepatocytes and serves as a chaperone capable of binding and transporting intestine-absorbed and metabolism-derived iron to other cells and tissues [10]. Its concentration in the serum is approximately $200-300 \mathrm{mg} / \mathrm{dl}$, but decreased levels are seen during inflammation and hepatic dysfunction [1, 7, 10, 11]. Particularly low serum transferrin values occur in individuals with sepsis or acute-on-chronic liver failure [7, 12, 13]. In critically ill patients and individuals with decompensated liver cirrhosis, severe alcoholic hepatitis (AH), or acute liver failure, low transferrin levels associate with increased mortality. Notably, this association is independent of established predictive scores such as Model for EndStage Liver Disease, markers of liver synthesis or inflammation [7, 12-17].

While transferrin represents an attractive adjunct to the existing prognostic scores, the biological mechanisms underlying its predictive power remain unclear. Here, we studied the mechanisms regulating transferrin production in individuals with advanced liver disease and $\mathrm{AH}$ and show that serum transferrin levels reflect the hepatic activity of HNF4 $\alpha$. Thus, transferrin constitutes both a prognostic and a mechanistic biomarker and an attractive surrogate for therapeutic trials aiming to preserve HNF4 $\alpha$ signaling as a tool to prevent the development of liver failure.

\section{Methods}

Human serum and liver specimens for biochemical analysis (cohort i)

Forty liver samples from patients who underwent a liver biopsy at the Universities of Aachen and Ulm between the years 2006 and 2018 were analyzed. The diagnosis of HCV was confirmed by a positive HCV RNA test, and all patients were treatment-naive. The diagnosis of ALD/ NAFLD was based on laboratory parameters, histological findings, personal interview, and an exclusion of other liver disease etiologies including viral infection, hereditary hemochromatosis, and autoimmune liver disease. NAFLD patients had characteristic histological findings without significant alcohol consumption (Table 1). Among them, a matched serum pair was available for 23 samples that were used for correlation analysis. Four liver samples from surrounding, non-affected tissue of liver metastases from patients without known chronic liver disease had been used as controls (Table 1). Staging of fibrosis was done on 4- $\mu$ m-thick liver sections using Desmet (HCV, controls) and Kleiner (ALD, NAFLD) scores. Publicly available data on transferrin levels in liver disease were obtained from GEO database and in silico analysis was performed on the GSE103580 dataset in 13 patients with alcoholic hepatitis [18]. The study was conducted in compliance with the Declaration of Helsinki (Hong Kong amendment) and Good Clinical Practice (European guidelines) as reflected in an approval by the local ethics committees of the participating centers. All participants, or their legally appointed representatives, provided written informed consent.

\section{Transcriptomic analysis in human liver specimens (cohort} ii)

RNA-seq was performed on liver tissue biopsies obtained from patients with early alcoholic steatohepatitis $(n=12)$, non-severe $\mathrm{AH}(n=11)$, severe $\mathrm{AH}(n=18)$, explants from patients transplanted with severe $\mathrm{AH}(n=$ 9), and normal background liver specimens from

Table 1 Patient's characteristics (cohort i)

\begin{tabular}{llll}
\hline Sample cohort & M/F & Age $^{*}$ & Fibrosis stage $^{*}$ \\
\hline Control $^{+}$ & $3 / 1$ & $61.2 \pm 16.6$ & $0 \pm 0$ \\
ALD & $10 / 4$ & $53.4 \pm 10.4$ & $3.6 \pm 0.5$ \\
NAFLD/NASH & $3 / 5$ & $58.1 \pm 11.6$ & $3.6 \pm 0.5$ \\
HCV & $7 / 3$ & $56.9 \pm 6.5$ & $4 \pm 0$ \\
Other etiologies $^{\#}$ & $2 / 6$ & $52.4 \pm 15.8$ & $4 \pm 0$
\end{tabular}

ALD alcoholic liver disease, HCV chronic hepatitis C infection, NAFLD nonalcoholic fatty liver disease, NASH non-alcoholic steatohepatitis

\#Other etiologies include one patient with chronic hepatitis B and ALD, one

with autoimmune hepatitis, one with cryptogenic cirrhosis, and one with biliary cirrhosis

${ }^{+}$Control samples were used for western blotting and staining

*Data are expressed as mean \pm SD 
patients resected for benign lesions $(n=10)$, from the InTEAM Consortium - Alcoholic Hepatitis Liver RNA Sequencing study, sponsored by the National Institute of Alcohol Abuse and Alcoholism (NIAAA, USA). The study details and sequencing data can be found in the Database of Genotypes and Phenotypes (dbGAP, phs001807.v1.p1) of the National Institutes of Health (NIH, USA). The basic clinical and laboratory data of the patients included in this study, the methods used to extract RNA and perform deep RNA sequencing, and the bioinformatic pipelines used to determine transcript counts have been described elsewhere [9]. In order to uncover the transferrin co-expressed transcriptome, the Kendall rank correlation coefficient was calculated. For pathway analysis, directly and reversely correlated genes with $p$ value inferior to $10^{-12}$ were selected. The Kendall coefficient ranges for selected genes was +0.76 to +0.91 for the directly correlated ( $n=309$ genes) and -0.76 to -0.84 for the reversely correlated ( $n=94$ genes). Ingenuity Pathway Analysis (IPA, Qiagen Inc., version 2017) was used to uncover main upstream regulators.

\section{Genomic DNA methylome analysis (cohort ii)}

Liver DNA methylation studies have been described previously [9]. Briefly, genomic DNA (gDNA) was extracted from flash-frozen liver tissue with PureLink Genomic DNA Mini Kit (Thermo) and quantified using Nanodrop (Thermo). One microgram of isolated gDNA was bisulfite converted, denatured, fragmented, and hybridized to Infinium Methylation Bead Chip, following the manufacturer's protocol (Infinium MethylationEPIC kit, Illumina). BeadChips were imaged using an Illumina Scan System, and intensity was determined by the iScan Control Software (Illumina). Sample intensities were normalized using functional normalization from the minfi package (v1.24.0) [19]. Probes failing a detection $p$ value threshold $(0.01)$ in at least $50 \%$ of samples were removed, as were probes identified as containing a SNP with a MAF $>0.05$. Differentially methylated probes were identified by applying limma (v3.34.3) contrasts to $M$ values (absolute change in beta value $>0.1$, FDRcorrected $p$ value $<0.05$ ) [20]. Differentially methylated regions were identified using DMRcate (v1.14.0) [21] setting a threshold of absolute change in beta value in $>0.1$ and of Stouffer's value in $<0.05$. The package gviz ( $\mathrm{v}$ 1.28.0) [22] was used to visualize transferrin genomic region, including methylation beta values. The information downloaded from the University of California Santa Cruz (UCSC) server of the Human Genome Sequencing Consortium included HepG2 HNF4A ChIP-seq BigWig data (Sample GSM803460 of series GSE32465) and DNAse I hypersensitive sites Bed data (wgEncodeRegDnaseClusteredV3.bed, UW and Duke ENCODE data).

\section{Chromatin immunoprecipitation and DNA sequencing} (ChIP-seq) of histone marks (cohort ii)

ChIP-seq was performed in Mayo Epigenomics Development Laboratory (EDL) [23] with the liver tissue from 5 controls and 7 severe AH explants (provided by University of Lille, France). It was carried out for four histone modifications, using antibodies against histone $\mathrm{H} 3$ Lysine 27 acetylation (H3K27ac, Cell Signaling \#8173), histone H3 Lysine 27 tri-methylation (H3K27me3, Cell Signaling \#9733), histone H3 Lysine 4 monomethylation (H3K4me1, EDL, Mayo Clinic, Lot\#1), and histone H3 Lysine 4 tri-methylation (H3K4me3, EDL, Mayo Clinic, Lot\#1). For the next-generation sequencing, ChIP-seq libraries were prepared from $10 \mathrm{ng}$ of ChIP and input DNAs with the Ovation Ultralow DR Multiplex System (NuGEN). The ChIP-seq libraries were sequenced to 51 base pairs from both ends using the Illumina HiSeq 2000 in the Mayo Clinic Medical Genomics Core. Data were analyzed by the HiChIP pipeline [24]. Briefly, reads were aligned to the hg19 genome assembly using BWA and visualized using the Integrative Genomics Viewer (IGV). Mapped reads were postprocessed to remove duplicates and pairs of reads mapping to multiple locations. The MACS2 and Sicer algorithm was used for peak calling in relation to the input DNA. In this study IGV was then used to visualize the peak changes on transferrin genomic region in this study.

\section{Biochemical analysis (cohort i)}

Four control and 6 cirrhotic samples were used for biochemical analysis. Tissue lysates were prepared in homogenization buffer containing 3\% sodium dodecyl sulphate (SDS) and were diluted with $4 \times$ reducing Laemmli buffer afterwards. Proteins were separated via 10\% SDS polyacrylamide gel electrophoresis and transferred to PVDF membranes (GE Healthcare/Amersham Biosciences, Germany) for immunoblotting. After incubation with the primary and horse radish peroxidase (HRP)-coupled secondary antibodies, the resulting HRP signal was detected with an ECL Detection kit (GE Healthcare/Amersham Biosciences, UK). The following antibodies were used: human anti-transferrin (Sigma; HPA005692, Stockholm, Sweden), mouse anti-transferrin (Abcam; Ab82411), anti-HNF4alpha (Sigma; HPA004712, Bromma Sweden), and anti-GAPDH (Novus; NB300-221, Nordenstadt, Germany).

\section{Tissue stainings (cohort i)}

For immunohistochemistry, 3-4- $\mu$ m-thick liver sections were deparaffinized and rehydrated, and a citrate-based antigen retrieval was performed (Vector H-3300, Vector Laboratories, Petersborough, UK) for $30 \mathrm{~min}$, as recommended by the supplier. The immunodetection was 
carried out with anti-transferrin as a primary $(11,000$ dilution; Sigma; HPA005692, Stockholm, Sweden) and a biotin-conjugated anti-rabbit secondary antibody (Vector BA1000). The signal was visualized with peroxidaselabeled streptavidin and the 3,3'-diaminobenzidine as a substrate (Vector SK4100).

\section{Measurement of serum parameters (cohort i)}

Serum transferrin (turbidimetry), iron, and ferritin levels were measured with routine assays using the Cobas 8000 system (Roche Diagnostics, Mannheim, Germany) available at the Clinical Chemistry Department of University Hospital Aachen. Serum apolipoprotein B (APOB; R\&D Systems DAPB00, Minneapolis, MN, USA), human tranthyretin (Prealbumin; Avivasysbio, OKIA00081, San Diego, CA, USA), and complement C3 (Abcam, ab108822, Berlin, Germany) concentrations were measured by using commercial sandwich ELISAs.

\section{Primary hepatocyte cell culture}

Primary hepatocytes were isolated from 3- to 4-monthold C57BL/6 mice as described previously [25]. Primary hepatocytes were kept in culture medium (DMEM; PAN Biotech, Aidenbach, Germany) supplemented with 10\% FBS, 1\% penicillin-streptomycin, and L-glutamine (PAN Biotech). After overnight incubation, the medium was replaced by William's E medium (PAN Biotech), and cells were treated for $6 \mathrm{~h}$ with IL- $1 \beta(20 \mathrm{ng} / \mathrm{ml}$; Peprotech, Hamburg, Germany), IL-6 (40 ng/ml; Sigma, St. Louis, MO, USA), TNF $\alpha$ (30 ng/ml; Peprotech), and the HNF4 $\alpha$ antagonist BI6015 (50 $\mu \mathrm{M}$; Millipore, Burlington, MA, USA) or for $24 \mathrm{~h}$ with TGF $\beta 1(10 \mathrm{ng} / \mathrm{ml}$; R\&D Systems, Minneapolis, MI, USA). Alternatively, the cells were transfected with the siRNA against HNF4 $\alpha(60$ pmol; Thermo Fisher Scientific ID: 158081 and 158082, Germany) according to the manufacturer's protocol (Lipofectamine $^{\bullet}$ RNAiMAX $^{\mathrm{su}}$ Transfection Reagent, Thermo Fisher Scientific, Germany) and cultured for 48 h. RNA was isolated via RNeasy tissue mini isolation kit (Qiagen, Hilden, Germany). The RNA samples were translated to cDNA with the M-MLV reverse transcriptase kit (Promega, Madison, WI, USA) and random hexamers (Thermo Scientific, Waltham, MA, USA). The relative expression of genes of interest was determined using specific primers (Additional file 1: Table S1). The mouse ribosomal gene L7 was used as an internal loading control.

\section{Data processing and statistical analysis}

Based on the results of a normality test, continuous variables were compared by an unpaired $t$ test. Correlations between clinical variables and serum proteins were tested by Spearman's rank correlation test. Nominal two-sided $p$ values were reported for all tests and were considered to be statistically significant when $p<0.05$ unless mentioned otherwise. Statistical analyses were performed, and graphs were created with GraphPad Prism (GraphPad, La Jolla, CA, USA).

\section{Results}

Patients with advanced liver disease display decreased hepatic transferrin expression

To delineate the factors underlying the predictive usefulness of transferrin in severe liver disease, we compared the hepatic expression of transferrin and transferrin serum levels in 23 patients with advanced liver disease and available matched mRNA-serum sample pairs (Table 1) (cohort i). Immunoblotting revealed decreased transferrin protein levels in cirrhotic livers (Fig. 1a, b). Accordingly, immunohistochemistry manifested a strong, homogeneous hepatocellular staining in control livers, whereas a patchy, diminished signal was seen in cirrhotic patients (Fig. 1c) (cohort i). In individuals with different stages of $\mathrm{AH}$ (cohort ii), a progredient decrease in normalized transferrin gene transcripts was seen at more severe stages (Fig. 1d). Spearman's correlation showed a moderate correlation between the serum transferrin levels and hepatic mRNA expression in individuals with advanced liver fibrosis and available matched serum-mRNA sample pairs (Fig. 1e) (cohort i). Notably, serum transferrin levels did not correlate with serum levels of other established hepatocellular products such as alpha1-antitrypsin, ceruloplasmin, albumin, or apolipoprotein B (not shown). On the other hand, in cohort (i), we observed a moderate negative correlation between serum ferritin and transferrin levels, while no correlation was seen between serum transferrin and serum iron levels (Additional file 1: Fig. S1).

\section{HNF4a regulates hepatocellular transferrin production}

To determine the factors responsible for altered hepatic transferrin expression, we analyzed RNA-seq data from healthy individuals as well as patients with different stages of alcohol-related liver disease (ALD). This cohort comprised the full spectrum of ALD, from early asymptomatic disease in heavy drinkers to patients with alcoholic hepatitis (Figs. 1d and 2a) (cohort ii). The expression of TF in the liver of patients with $\mathrm{AH}$ was significantly reduced in patients with severe $\mathrm{AH}$ (Fig. 1d). We then performed a gene-gene correlation analysis of TF expression with the whole transcriptome, stratifying by disease severity (Fig. 2a). The most correlated genes were then used to infer transcription factor footprint using Ingenuity Pathway Analysis. The analysis of upstream regulators revealed HNF4 $\alpha$ and TGF $\beta 1$ as the factors that are most tightly linked to the transferrin-correlated 


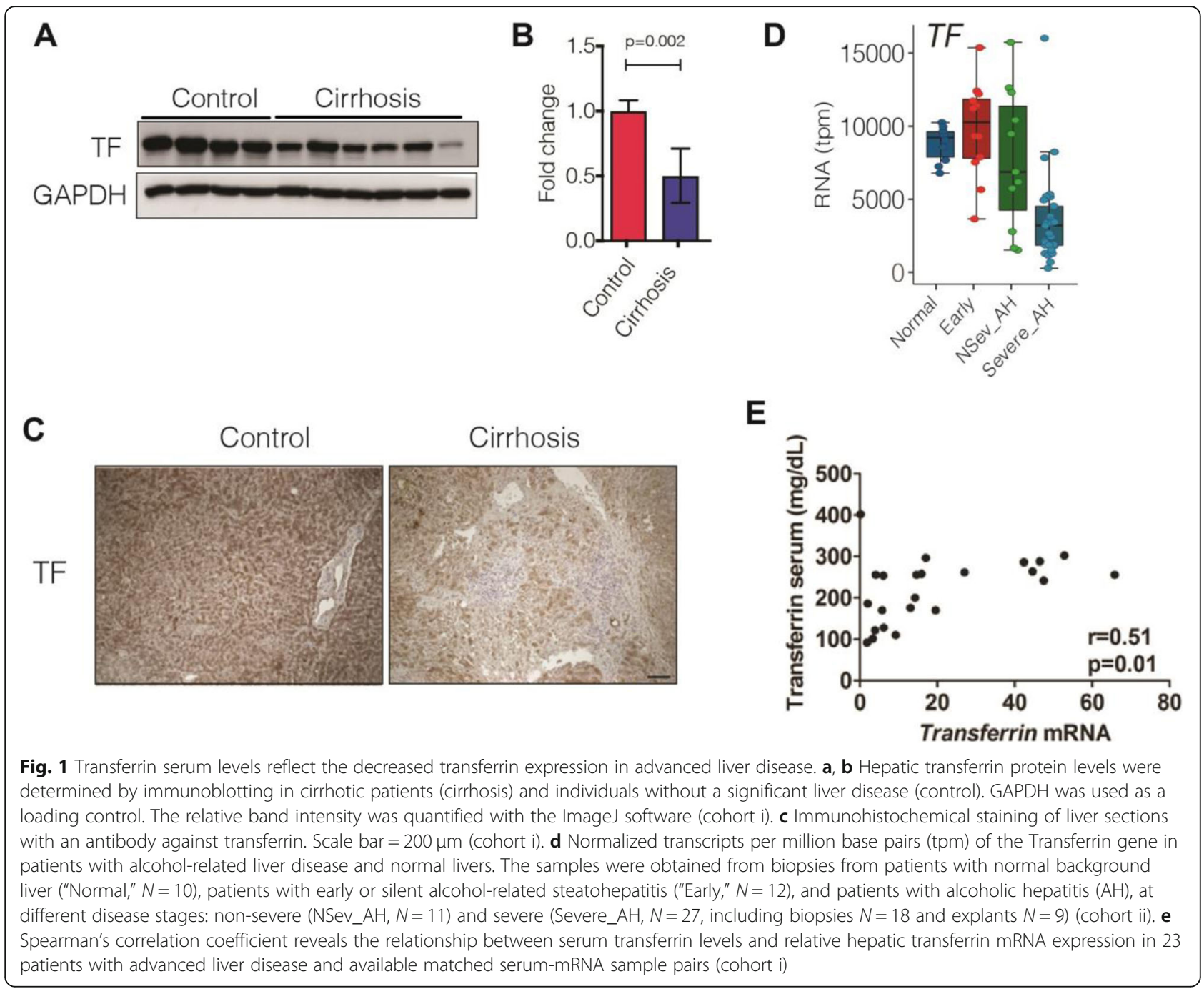

transcriptome (Fig. 2b, c) (cohort ii). This result suggests that transferrin expression could be a bona fide marker of hepatic HNF4 $\alpha$ downregulation. The established drivers of acute phase response, i.e., TNF $\alpha$, IL$1 \beta$, and IL- 6 also displayed a strong negative correlation with transferrin mRNA in our upstream regulation analysis (Fig. 2b). Since these analyses were made using whole liver RNA, the impact of infiltrating nonparenchymal cells on these transcriptomic footprints cannot be excluded.

\section{Epigenetic and molecular regulation of hepatic transferrin expression}

To further understand the regulation of transferrin in patients with $\mathrm{AH}$, we analyzed the transferrin genomic region using liver methylomic data as well as chromatin immunoprecipitation coupled to DNA sequencing data (ChIP-Seq) from a previous study in patients with different stages of alcoholic liver disease (Figs. 3 and 4) [9] (cohort ii). We compared these genomic regions with different epigenetic marks and HNF4A ChIP-seq available in ENCODE. The methylome analysis revealed a differentially methylated region in a regulatory region of the transferrin promoter, which was bound by HNF4 $\alpha$. Patients with AH had higher levels of methylation in different regulatory $\mathrm{CpG}$ islands in this region, indicating a possible cause of transferrin downregulation (Fig. 3). In a different set of $\mathrm{AH}$ patients, ChIP-Seq showed increased levels of H3K27me3 mark and a decrease on H3K4me3 and H3K27ac marks in the same region, indicating that the chromatin conformation promotes repression of transferrin gene expression in these patients (Fig. 4). To test whether these associations imply a direct regulation, we used primary mouse hepatocytes (Fig. 5a). In these, TGF $\beta 1$ treatment markedly suppressed transferrin expression (Fig. 5b) whereas IL-1 $\beta$, TNF $\alpha$, and IL6 treatment did not have a significant effect (Fig. 5c-e). Treatment with the HNF4 $\alpha$ antagonist BI6015 resulted in an almost complete suppression of transferrin expression (Fig. 5f), while no significant alterations were seen 


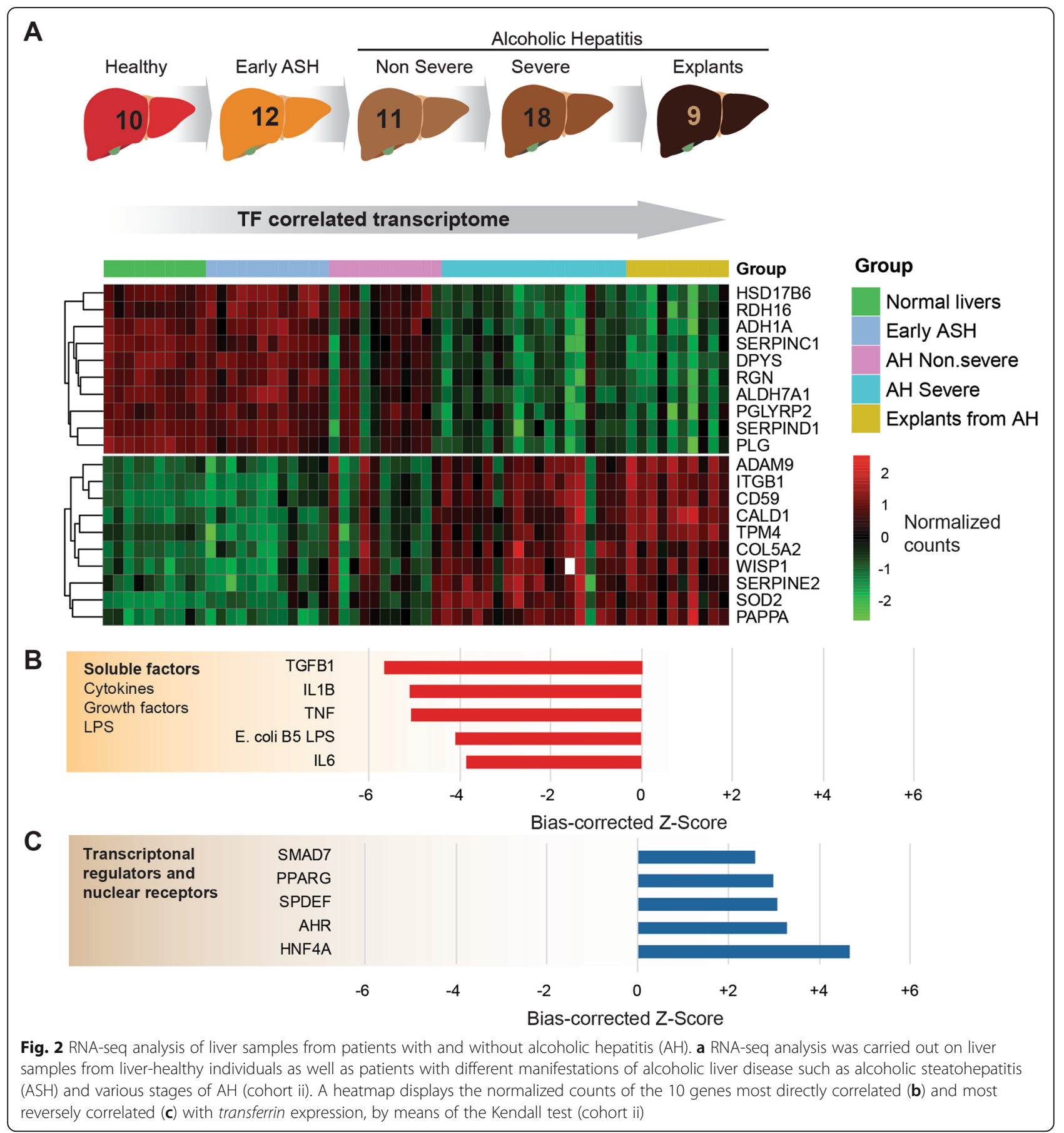

in HNF4 $\gamma$ levels that are known to be affected by high doses of BI6015 (not shown). RT-PCR for HNF4A, which was used to confirm the efficacy of the described treatments, revealed the suppression of HNF4 $\alpha$ expression in both the antagonist- and TGF $\beta 1$-treated hepatocytes (Fig. 5b, f). Finally, a treatment with HNF4A siRNA resulted in an almost complete suppression of HNF4A mRNA levels and significantly reduced transferrin protein levels (Additional file 1: Fig. S2).

\section{Transferrin is superior to other genes as a surrogate} marker of HNF4a activity

Finally, to further corroborate the correlation between HNF4A and transferrin expression, we analyzed publicly available data on 13 patients with AH (GSE103580) [18]. In these individuals, we observed a strong association between hepatic transferrin and HNF4A levels $(r=0.70$ $p=0.007$; Additional file 1: Fig. S3A). A comparably strong correlation was seen in 40 patients with advanced 


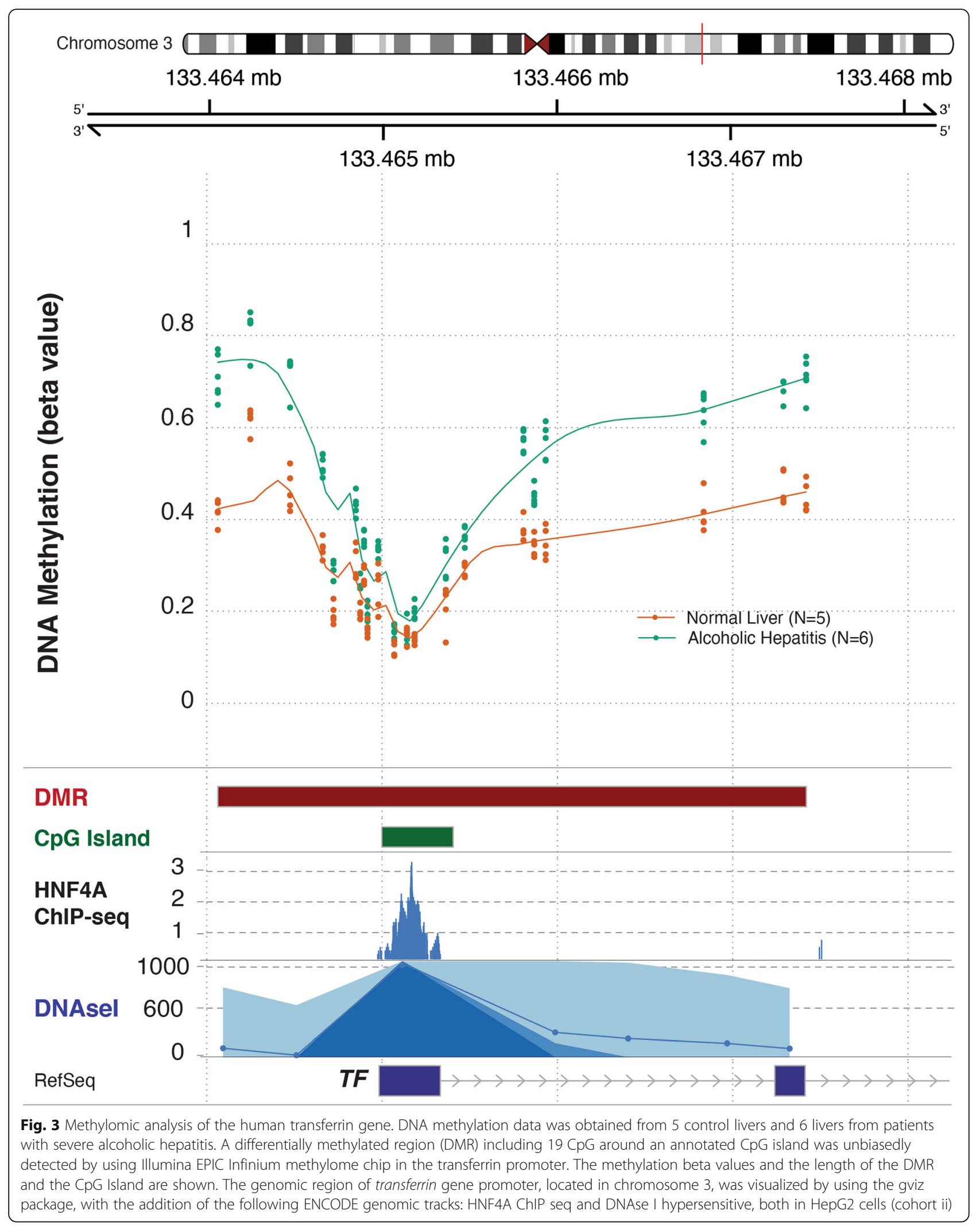




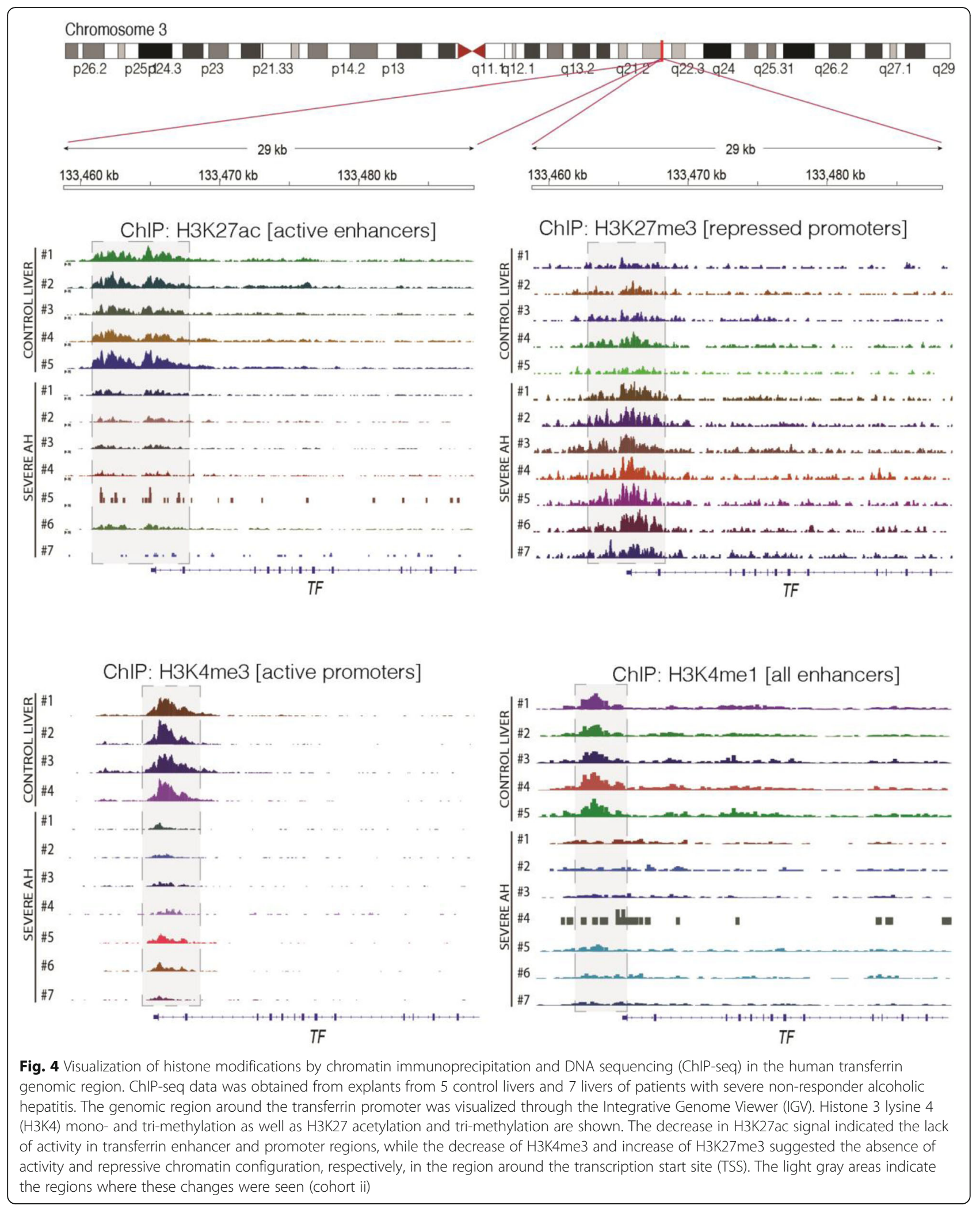

liver disease $(r=0.59 p<0.0001$; Additional file 1: Fig. S3B). These data strongly suggest that transferrin decrease reflects an impaired HNF4 $\alpha$ axis. In line with that, immunoblotting demonstrated decreased HNF4 $\alpha$ protein levels in cirrhotic vs. control livers (Additional file 1: Fig. S3C). 


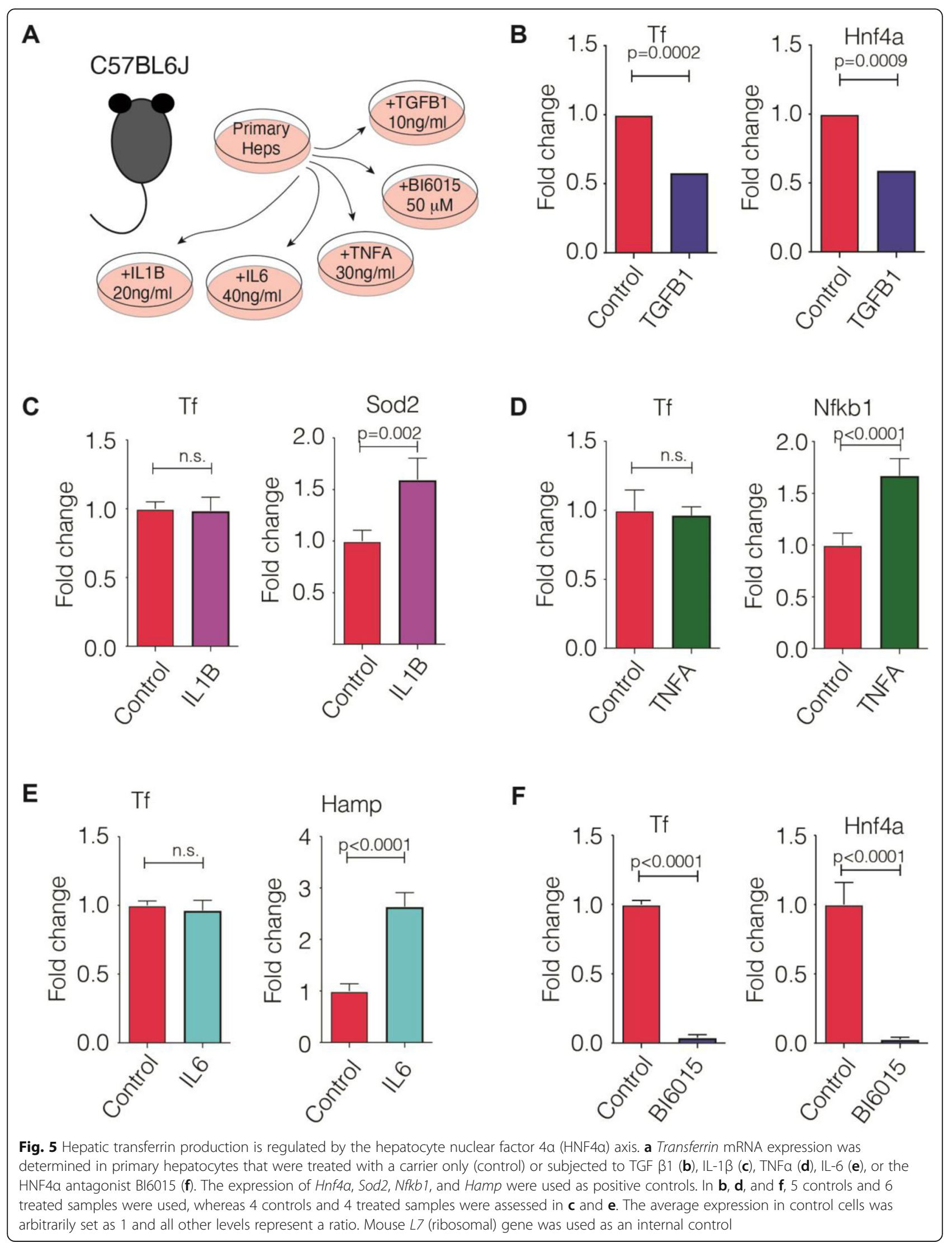


To identify additional markers of impaired HNF4 $\alpha$ signaling, we assessed three established HNF4 $\alpha$ target genes, i.e., apolipoprotein B (ApoB), transthyretin (TTR), and complement C3 (C3). Spearman's correlation coefficient revealed a moderate correlation between the mRNA levels of HNF4A and its targets ApoB and C3 but no significant correlation with TTR (Additional file 1: Fig. S4). Moreover, in none of the analyzed species, a significant correlation between serum and hepatic mRNA was detected (Additional file 1: Fig. S4). Notably, similar results were seen when only subjects with ALD/NAFLD were included in the analysis (not shown). Collectively, our data indicate that transferrin may constitute an attractive serological marker reflecting the hepatic HNF4 $\alpha$ signaling.

\section{Discussion}

In our study, we systematically analyzed the factors regulating the serum transferrin levels and demonstrated that serum transferrin levels correlate with hepatic transferrin production and that hepatic transferrin production is not only directly regulated by, but also reflects, the activity of HNF4 $\alpha$ signaling. The former findings are well in line with the study from Potter et al., who analyzed transferrin synthesis and serum levels in individuals with alcoholic liver disease [11]. The finding that transferrin mRNA levels strongly correlate with hepatic HNF4 $\alpha$ levels is compatible with the data from liver-specific HNF4 $\alpha$ knockout mice, which displayed a deranged iron metabolism and a diminished transferrin expression [26]. Notably, transferrin expression is not altered after exposure to TNF $\alpha$, IL-1 $\beta$, and IL- 6 that constitute the major regulators of hepatocellular acute phase response $[1$, 6]. While we focused on $\mathrm{AH}$ and advanced liver disease, the decreased transferrin levels seen in individuals with acute liver failure and in critically ill patients [7, 14] suggest that similar mechanisms may apply to multiple different stress situations. This is of particular interest, since no correlation between hepatic expression and serum protein levels was seen for three other well-known HNF4 $\alpha$ targets. However, our study has also important limitations. First, it primarily focused on alcoholic liver disease, and further studies are needed to determine to what extent our findings apply to other disease etiologies. Additionally, the impact of hepatic iron load on serum transferrin levels needs to be assessed.

Our data demonstrating that transferrin levels indicate the activity of the HNF4 $\alpha$ axis is of obvious relevance. HNF4 $\alpha$ constitutes a major liver-enriched transcription factor, that is responsible for the production of multiple mature hepatocyte-specific genes [26, 27]. Moreover, HNF4 $\alpha$ activity is diminished in multiple liver disorders including $\mathrm{AH}$ and decompensated liver cirrhosis and is likely crucial for their pathogenesis [2, 9]. In line with that, a forced re-expression of HNF4 $\alpha$ rapidly reversed fatal liver failure in CCl4-treated rats [28]. In agreement with our data, the downregulation of HNF4 $\alpha$ signaling in $\mathrm{AH}$ occurs via TGF $\beta$ signaling and is thought to be mediated through DNA methylation and chromatin remodeling $[9,29]$. On the other hand, the regulation of HNF $4 \alpha$ signaling and the role of methylation remain to be systematically analyzed. Notably, a smaller study reported a correlation between serum levels of transferrin and TGF $\beta$ in patients admitted to an internal medicine department [30]. While it is tempting to speculate that decreased transferrin levels are induced via TGF $\beta$-induced, epigenetic changes in transferrin production, further studies are needed to corroborate this hypothesis.

The observation that serum transferrin is both a prognostic and a mechanistic biomarker may have direct medical implications. As such, transferrin levels can be used for patient stratification and treatment allocation in targeted clinical trials aiming to enhance HNF4 $\alpha$ signaling. In that respect, the FDA-approved PPARy agonist rosiglitazone was suggested as a potential candidate for the treatment of liver failure [9]. Transferrin represents an attractive surrogate of mortality in such trials. Second, lowered transferrin levels may not be only an indicator of imminent liver failure, but also a promising candidate for plasma-based therapeutic interventions. While administration of transferrin yielded promising results in various clinical conditions such as atransferrinemia and iron overload or in animal models of $\beta$ thalassemia [31,32], it was never tested in the context of liver disease. This approach might be particularly attractive given the emerging therapeutic value of plasma exchange and/or the well-documented usefulness of plasma products such as albumin in the treatment of both acute and chronic liver failure [33, 34].

\section{Conclusions}

Our study reports that transferrin is a novel serological surrogate of impaired HNF4 $\alpha$ axis and as such might be useful both for prognostic evaluation as well as to direct and stratify patients for novel therapeutic approaches. Prospective studies are warranted to corroborate the relationship between HNF4 $\alpha$ and transferrin levels in several forms of liver failure, i.e., acute liver failure, acute-on-chronic liver failure, and $\mathrm{AH}$.

\section{Supplementary Information}

The online version contains supplementary material available at https://doi. org/10.1186/s12916-021-01917-6.

\footnotetext{
Additional file 1 : Figure S1-S4. Figure S1 Correlation between serum parameters of iron metabolism in samples from cohort (i). Figure $\mathbf{S 2}$ Hepatic transferrin production is regulated by the hepatocyte nuclear factor $4 a(H N F 4 a)$ axis. Figure $\mathbf{S 3}$ Transferrin expression correlates with HNF4a expression in liver disease. Figure S4 Correlation of selected HNF4a targets with the hepatic HNF4a expression in patients with advanced liver disease (cohort i). Table S1 Primer sequences used for experiments in primary hepatocyte culture.
} 


\section{Abbreviations}

AH: Alcoholic hepatitis; ALD: Alcohol-related liver disease;

APOB: Apolipoprotein B; APPs: Acute-phase proteins; ASH: Alcoholic steatohepatitis; C3: Complement C3; CCl4: Carbon tetrachloride; ChIPSeq: Chromatin immunoprecipitation coupled to DNA sequencing data; CRP: C-reactive protein; dbGAP: Database of Genotypes and Phenotypes; GAPDH: Glyceraldehyde-3-phosphate dehydrogenase; HAMP: Hepcidin antimicrobial peptide; HCV: Chronic hepatitis C infection; HNF4a: Hepatocyte nuclear factor 4 alpha; IL-1 $\beta$ : Interleukin 1 beta; IL-6: Interleukin 6; L7: Large ribosomal subunit; NAFLD: Non-alcoholic fatty liver disease; NASH: Nonalcoholic steatohepatitis; NFKB1: Nuclear factor of kappa light polypeptide gene; PPARY: Peroxisome proliferator-activated receptor $\gamma$; RPLPO: Ribosomal protein lateral stalk subunit P0; SOD2: Superoxide dismutase 2; TF: Transferrin TGF $\beta 1$ : Transforming growth factor beta 1; TNFa: Tumor necrosis factor alpha; TTR: Transthyretin

\section{Acknowledgements}

We thank all patients for their participation in our study.

\section{Further remarks}

We attest that we did not use any copyright protected material in our manuscript. No writing assistance was provided.

\section{Authors' contributions}

Study concept and design: N.G., S.R.A., M.R.T., R.B., and P.S. Acquisition of the data: N.G., J.A., B.G., S.R.A., R.B., P.S., J.J.L., J.M., and T.B. Analysis and interpretation of the data: N.G., J.A., S.R.A., R.B., and P.S. Drafting of the manuscript: N.G. and P.S. Critical revision of the manuscript for important intellectual content: all authors. Statistical analysis: N.G. and J. A. Figures and tables: N.G., J. A., and P.S. Obtained funding: J.A., S.R.A., M.R.T., J.M., R.B., N.G., C.T., T.B., and P.S. Study supervision: N.G., J.A., S.R.A., M.R.T., R.B., and P.S. All authors had full access to all of the data. All authors read and approved the final manuscript. All authors can take responsibility for the integrity of the data and the accuracy of the data analysis.

\section{Funding}

This work was supported by the Deutsche Forschungsgemeinschaft (DFG) grants SFB/TRR57, SFB 1382 (ID 403224013) (to P.S., N.G., T.B., and C.T.), and STR 1095/6-1 (Heisenberg professorship to P.S.); the Medical Research Council (to S.R.A. and M.R.T. (Grant MR/R014019/1), to J.M. (Grant MR/ K10019494/1)); and the National Institute for Health Research (to M.R.T.) S.R.A. and M.R.T. acknowledge the support of the NIHR Imperial Biomedical Research Centre. R.B. was supported by the following grants: U01AA026978, U01AA026972, and 1P30DK120531-01. Open Access funding enabled and organized by Projekt DEAL.

\section{Availability of data and materials}

The datasets used and analyzed during the current study are available from the corresponding author on reasonable request.

\section{Ethics approval and consent to participate}

For all cohorts, ethics approval and informed consent to participate in the study are available (cohort i: Institutional review board of Aachen and UIm universities, cohort ii: for human RNA-seq studies, human liver samples were obtained from the Human Biorepository Core from the NIH-funded international InTeam consortium (7U01AA021908-05). Patients with early alcoholic steatohepatitis (ASH) were obtained from Cliniques Universitaires Saint-Luc (Brussels, Belgium). All patients (or their legal representatives) gave written informed consent, and the research protocols were approved by the local Ethics Committees and by the central Institutional Review Board of the University of North Carolina at Chapel Hill [9].

\section{Consent for publication}

Not applicable.

\section{Competing interests}

All authors declare that no support from any organization other than the below-mentioned ones for the submitted work, no financial relationships with any organizations that might have an interest in the submitted work in the previous 3 years; and no other relationships or activities that could appear to have influenced the submitted work. Hence, all authors declare themselves to be independent from funders with respect to this manuscript.

\section{Author details}

${ }^{1}$ Department of Internal Medicine III, University Hospital RWTH Aachen, Pauwelsstraße 30, 52074 Aachen, Germany. ${ }^{2}$ Pittsburgh Liver Research Center, University of Pittsburgh Medical Center, Pittsburgh, PA, USA. ${ }^{3}$ Liver Unit, Clinica Universidad de Navarra, Hepatology Program, Center for Applied Medical Research, Pamplona, Spain. ${ }^{4}$ Department of Hepatology, Imperial College London, London, UK. ${ }^{5}$ Center of Cardiovascular Surgery and Transplantation Brno, Brno, Czech Republic. ${ }^{6}$ Research Institute Valdecilla (Instituto de Investigación Sanitaria Valdecilla), Santander, Spain.

${ }^{7}$ Gastroenterology and Hepatology Unit, University Hospital Marqués de Valdecilla, Santander, Spain. ${ }^{8}$ Centro de Investigacion Biomedica en Red, Enfermedades Hepáticas y Digestivas (CIBERehd), Barcelona, Spain.

${ }^{9}$ Newcastle Fibrosis Research Group, Biosciences Institute, Faculty of Medical Sciences, Newcastle University, Newcastle, UK. ${ }^{10}$ Division of Gastroenterology and Hepatology, Mayo Clinic, Rochester, MN, USA. ${ }^{11}$ Hôpital Claude Huriez, Services des Maladies de l'Appareil Digestif, CHRU Lille, and Unité INSERM 995, Lille, France.

Received: 30 June 2020 Accepted: 18 January 2021 Published online: 17 February 2021

\section{References}

1. Kuscuoglu D, Janciauskiene S, Hamesch K, Haybaeck J, Trautwein C, Strnad P. Liver - master and servant of serum proteome. J Hepatol. 2018;69(2):51224.

2. Guzman-Lepe J, Cervantes-Alvarez E, Collin del 'hortet A, Wang Y, Mars WM, Oda $Y$, et al. Liver-enriched transcription factor expression relates to chronic hepatic failure in humans. Hepatol Commun. 2018;2(5):582-94.

3. Schrem H, Klempnauer J, Borlak J. Liver-enriched transcription factors in liver function and development. Part I: the hepatocyte nuclear factor network and liver-specific gene expression. Pharmacol Rev. 2002;54(1):129-58.

4. Strnad P, Tacke F, Koch A, Trautwein C. Liver - guardian, modifier and target of sepsis. Nat Rev Gastroenterol Hepatol. 2017;14(1):55-66.

5. Ritchie RF, Palomaki GE, Neveux LM, Navolotskaia O. Reference distributions for the negative acute-phase proteins, albumin, transferrin, and transthyretin: a comparison of a large cohort to the world's literature. J Clin Lab Anal. 1999;13(6):280-6.

6. Gabay C, Kushner I. Acute-phase proteins and other systemic responses to inflammation. N Engl J Med. 1999;340(6):448-54.

7. Tacke F, Nuraldeen R, Koch A, Strathmann K, Hutschenreuter G, Trautwein C et al. Iron parameters determine the prognosis of critically ill patients. Crit Care Med. 2016:44(6):1049-58.

8. Akdemir V, Sut N, Guldiken B. Factors affecting the quality of life in drugresistant epilepsy patients. Acta Neurol Belg. 2016;116(4):513-8.

9. Argemi J, Latasa MU, Atkinson SR, Blokhin IO, Massey V, Gue JP, et al. Defective HNF4alpha-dependent gene expression as a driver of hepatocellular failure in alcoholic hepatitis. Nat Commun. 2019;10(1):3126.

10. Kawabata H. Transferrin and transferrin receptors update. Free Radic Biol Med. 2019;133:46-54.

11. Potter BJ, Chapman RW, Nunes RM, Sorrentino D, Sherlock S. Transferrin metabolism in alcoholic liver disease. Hepatology. 1985;5(5):714-21.

12. Maras JS, Maiwall R, Harsha HC, Das S, Hussain MS, Kumar C, et al. Dysregulated iron homeostasis is strongly associated with multiorgan failure and early mortality in acute-on-chronic liver failure. Hepatology. 2015;61(4): 1306-20.

13. Bruns T, Nuraldeen R, Mai M, Stengel S, Zimmermann HW, Yagmur E, et al. Low serum transferrin correlates with acute-on-chronic organ failure and indicates short-term mortality in decompensated cirrhosis. Liver Int. 2017; 37(2):232-41.

14. Anastasiou OE, Kalsch J, Hakmouni M, Kucukoglu O, Heider D, Korth $J$, et al. Low transferrin and high ferritin concentrations are associated with worse outcome in acute liver failure. Liver Int. 2017; 37(7):1032-41.

15. Viveiros A, Finkenstedt A, Schaefer B, Mandorfer M, Scheiner B, Lehner K, et al. Transferrin as a predictor of survival in cirrhosis. Liver Transpl. 2018; 24(3):343-51.

16. Meier JA, Bokemeyer A, Cordes F, Fuhrmann V, Schmidt H, HüsingKabar A, Kabar I. Serum levels of ferritin and transferrin serve as 
prognostic factors for mortality and survival in patients with end-stage liver disease: A propensity score-matched cohort study. United European Gastroenterol J. 2020;8(3):332-9. https://doi.org/10.1177/ 2050640619891283. Epub 2019 Nov 26. PMID: 32213016; PMCID: PMC7184660

17. Atkinson SR, Hamesch K, Spivak I, Guldiken N, Cabezas J, Argemi J, et al. Serum transferrin is an independent predictor of mortality in severe alcoholic hepatitis. Am J Gastroenterol. 2020;115(3):398-405.

18. Trepo E, Goossens N, Fujiwara N, Song WM, Colaprico A, Marot A, et al. Combination of gene expression signature and model for end-stage liver disease score predicts survival of patients with severe alcoholic hepatitis. Gastroenterology. 2018;154(4):965-75.

19. Aryee MJ, Jaffe AE, Corrada-Bravo H, Ladd-Acosta C, Feinberg AP, Hansen $K D$, et al. Minfi: a flexible and comprehensive Bioconductor package for the analysis of Infinium DNA methylation microarrays. Bioinformatics. 2014; 30(10):1363-9.

20. Ritchie ME, Phipson B, Wu D, Hu Y, Law CW, Shi W, et al. limma powers differential expression analyses for RNA-sequencing and microarray studies. Nucleic Acids Res. 2015;43(7):e47.

21. Peters TJ, Buckley MJ, Statham AL, Pidsley R, Samaras K, RVL, et al. De novo identification of differentially methylated regions in the human genome. Epigenet Chromatin. 2015;8:6.

22. Hahne F, Ivanek R. Visualizing genomic data using Gviz and Bioconductor. Methods Mol Biol. 2016;1418:335-51.

23. Zhong J, Ye Z, Lenz SW, Clark CR, Bharucha A, Farrugia G, et al. Purification of nanogram-range immunoprecipitated DNA in ChIP-seq application. BMC Genomics. 2017;18(1):985.

24. Yan H, Evans J, Kalmbach M, Moore R, Middha S, Luban S, et al. HiChIP: a high-throughput pipeline for integrative analysis of ChIP-Seq data. BMC Bioinformatics. 2014;15:280

25. Guldiken N, Kobazi Ensari G, Lahiri P, Couchy G, Preisinger C, Liedtke C, et al. Keratin 23 is a stress-inducible marker of mouse and human ductular reaction in liver disease. J Hepatol. 2016:65(3):552-9.

26. Matsuo S, Ogawa M, Muckenthaler MU, Mizui Y, Sasaki S, Fujimura T, et al. Hepatocyte nuclear factor 4alpha controls iron metabolism and regulates transferrin receptor 2 in mouse liver. J Biol Chem. 2015; 290(52):30855-65.

27. Lau HH, Ng NHJ, Loo LSW, Jasmen JB, Teo AKK. The molecular functions of hepatocyte nuclear factors - in and beyond the liver. J Hepatol. 2018;68(5): 1033-48.

28. Nishikawa T, Bell A, Brooks JM, Setoyama K, Melis M, Han B, et al. Resetting the transcription factor network reverses terminal chronic hepatic failure. $J$ Clin Invest. 2015;125(4):1533-44.

29. Cardenas H, Vieth E, Lee J, Segar M, Liu Y, Nephew KP, et al. TGF-beta induces global changes in DNA methylation during the epithelial-tomesenchymal transition in ovarian cancer cells. Epigenetics. 2014;9(11): 1461-72.

30. Koorts AM, Levay PF, Becker PJ, Viljoen M. Pro- and anti-inflammatory cytokines during immune stimulation: modulation of iron status and red blood cell profile. Mediat Inflamm. 2011:2011:716301.

31. Li H, Rybicki AC, Suzuka SM, von Bonsdorff L, Breuer W, Hall CB, et al. Transferrin therapy ameliorates disease in beta-thalassemic mice. Nat Med. 2010;16(2):177-82

32. Boshuizen $M$, van der Ploeg $K$, von Bonsdorff L, Biemond BJ, Zeerleder SS, van Bruggen $R$, et al. Therapeutic use of transferrin to modulate anemia and conditions of iron toxicity. Blood Rev. 2017;31 (6):400-5.

33. Larsen FS, Schmidt LE, Bernsmeier C, Rasmussen A, Isoniemi H, Patel VC, et al. High-volume plasma exchange in patients with acute liver failure: an open randomised controlled trial. J Hepatol. 2016;64(1):69-78.

34. Bernardi M, Angeli P, Claria J, Moreau R, Gines P, Jalan R, Caraceni P, Fernandez J, Gerbes AL, O'Brien AJ, Trebicka J, Thevenot T, Arroyo V. Albumin in decompensated cirrhosis: new concepts and perspectives. Gut. 2020;69(6):1127-38. https://doi.org/10.1136/gutjnl-2019-318843. Epub 2020 Feb 26. PMID:32102926; PMCID: PMC7282556.

\section{Publisher's Note}

Springer Nature remains neutral with regard to jurisdictional claims in published maps and institutional affiliations.

\section{Ready to submit your research? Choose BMC and benefit from:}

- fast, convenient online submission

- thorough peer review by experienced researchers in your field

- rapid publication on acceptance

- support for research data, including large and complex data types

- gold Open Access which fosters wider collaboration and increased citations

- maximum visibility for your research: over $100 \mathrm{M}$ website views per year

At BMC, research is always in progress.

Learn more biomedcentral.com/submissions 